\title{
LINGUISTIQUE
}

\author{
MIGUEL ALBI APARICIO
}

Universidad de Leipzig

\section{LOS MODIFICADORES VERBALES; CONCEPTO Y CLASIFICACIÓN DESDE UN MODELO REVISADO DE VALENCIA LÓGICO-SEMÁNTICA}

\begin{abstract}
A bstract. Albi Aparicio Miguel, Los modificadores verbales; concepto y clasificación desde un modelo revisado de valencia lógico-semántica [Verbal modifiers, concept and dassification from a revised model of logico-semantic valency]. Studia Romanica Posnaniensia, Adam Mickiewicz University Press, Poznań, vol. XXXI: 2004, pp. 121-127. ISBN 83-232-1353-4, ISSN 0137-2475.

In this article a possible definition and classification of the verbal modifiers is proposed. The theoretical frame is the Valence Theory complemented with aspects from cognitive linguistics inspired by the 2 Level-Semantics. The classification is done on the basis of verbal semantics focussing on which entities are modified.
\end{abstract}

\section{LA VALENCIA LÓGICO-SEMÁNTICA Y LOS DOS NIVELES}

Como introducción al modelo de Teoría de Valencias queremos apuntar que seguimos la línea de investigación que inició Wilhelm Bondzio a finales de los años 60 con la llamada Valencia Lógico-Semántica y que de alguna manera adoptó y adaptó Gerd Wotjak. Dentro de este modelo de valencia, lo semántico ocupa el primer plano, a diferencia de otros surgidos también en esta época, en los que, siguiendo la tradición de Tesnière, tienen más peso los aspectos sintácticos.

Como su propio nombre indica, se parte de la existencia de dos niveles implicados en la formación e interpretación del significado de las expresiones linguísticas. Esto es algo que tiene en común con otras teorías que se han ido desarrollando posteriormente, de las que especialmente nos interesan las que se incluyen en el paradigma cognitivo. 
La existencia de al menos dos tipos de relaciones de los predicados verbales con los elementos que los acompañan en los usos oracionales es algo que se ha extendido en los modelos de valencia no sólo basados en la semántica. De las distintas denominaciones que se le han dado a este fenómeno he adoptado la de Bondzio (1974) de Valencia de Primer y Segundo Grado. La de primer grado (en adelante VG1) abarca a los predicados verbales con sus slots argumentales y su correspondencia como actantes. La de segundo grado (VG2) abarca la relación de los predicados con los modificadores que potencialmente lo pueden acompañar en la oración en función de adjuntos modales, temporales y locales. Lo que nos diferencia respecto a Bondzio es que partimos de la información contenida en dichos predicados verbales para ver cuáles de esos adjuntos los pueden acompañar en sus usos oracionales. La VG2 se ocupa exclusivamente de aquellos cuya aparición se puede predecir desde la información que proporciona el verbo, que creemos que abarca cómo máximo a los adjuntos que saturan su argumento referencial o situacional, en el ${ }^{1}$ caso de tenerlo, por lo que los conocidos como adverbios de oración quedan automáticamente excluidos. Esta valencia para los adjuntos se apoya en el fenómeno de la tipicalidad, introducido por Meinhard (1994: 75), inspirado en la semántica de prototipos.

En Berlín, Manfred Bierwisch y Ewald Lang proponen en los años 80 un marco teórico que ha sido adoptado y desarrollado por numerosos investigadores: es la llamada Semántica de Dos Niveles. Esta corriente adopta un marco cognitivo que tiene en cuenta la existencia de un nivel conceptual (en adelante CS), virtualmente universal o válido para todas las lenguas pertenecientes a culturas que presenten un nivel de desarrollo similar, tanto técnico como de la vida cotidiana. También se parte de la existencia de un nivel semántico (en adelante SF), particular de cada lengua, que es el encargado de dotar a estos conceptos de las peculiaridades tanto puramente linguiísticas como extralingüísticas propias de esa cultura.

El SF abarca exclusivamente la parte del significado de una expresión lingüística determinada por la gramática, su aportación es invariable, independientemente del contexto en que aparezca, como refleja Claudia Maienborn (1998: 2) con la cláusula: "One Form, One Meaning". Simplificando el modelo, se puede decir que el saber enciclopédico almacenado en la CS y su interacción con el $\mathrm{SF}$ es lo que garantiza la formación y comprensión correcta de los usos oracionales concretos, dotando al SF del conocimiento de la situación, del contexto y del mundo necesarios para comprender correctamente un uso concreto.

Aunque las dos teorías difieren en cuanto a la metodología y a la finalidad que persiguen, creemos que la combinación de ambas permite aprovechar las ventajas de una y otra.

' Los llamados ILP (Individual-level-predicates), como kosten (costar), ähneln (parecerse)..., al carecer de dicho argumento, no pueden ser modificados por estos elementos (Maienborn, 1998: 23). 


\section{LOS MODIFICADORES VERBALES}

En los niveles conceptual y semántico se trata de hacer una descomposición lo más detallada posible del significado de los conceptos y significados lingüísticos en cuestión. Serán estructuras de funtores, argumentos y modificadores. Los funtores son también llamados predicados básicos porque no se pueden descomponer más, son primitivos semánticos. Son constantes en el sentido de que siempre significan lo mismo, pero pueden exigir distinto número de argumentos y de distinta naturaleza, p.ej.: CAUSE - precisa un individuo o una proposición como primer argumento (externo) y una proposición como segundo (interno)-; HAVE (poseer: hiperónimo) - precisa un individuo como primer y segundo argumento-; ADESSE (encontrarse en) - individuo o proposición y localización-; INST (relaciona una variable situacional con una proposición).

Los argumentos son variables que representan individuos del tipo: $\mathrm{x}, \mathrm{y}, \mathrm{z} \ldots$ Los situacionales se representan con variables: $\mathrm{s}$, e..., dependiendo de si se trata de una situación, un evento, etc. También hay slots argumentales ocupados por predicados que se representan con variables: $P, Q$, etc., como es el caso de las caracterizaciones modales, locales o temporales exigidas por el verbo.

Además de funtores y argumentos, como ya he dicho, en estos niveles aparecen también modificadores, que pueden estar representados por las variables arriba mencionadas: P, Q, etc. o, si se trata de TEMP, en algunos casos por sucesivas localizaciones temporales adosadas a las proposiciones que modifican: $\left[(\ldots) \mathrm{t}_{\mathrm{i}} \&(\ldots) \mathrm{t}_{\mathrm{i}+\mathrm{k}} \&(\ldots) \mathrm{t}_{\mathrm{n}}\right]$.

Bondzio (1971: 93), en su descomposición del significado concibe a los modificadores como los elementos que modifican a los predicados básicos o funtores; son para él los responsables de las diferencias semánticas irrelevantes para la valencia que se dan dentro de los verbos pertenecientes a un mismo campo semántico. Como ejemplos utiliza por un lado los verbos töten (matar), ermorden (asesinar) y schlachten (sacrificar animales) y geben (dar), leihen (prestar) y schenken (regalar), por otro.

Algunos años más tarde cambia su concepto (Bondzio, Göllmer, 1976: 700): los modificadores pueden afectar tanto a los funtores como a los slots de la estructura semántica, además hay modificadores que afectan a la totalidad del significado, sobre todo concernientes al estilo. Esto supone una ampliación considerable del concepto, de manera que da entrada a elementos que a nuestro entender están situados fuera de la semántica verbal en sentido estricto. En su definición de 1976 también se incluyen modificadores de NPs o de oración, y estos elementos no son objeto de este estudio.

Maienborn (1990: 33) considera la modificación como la identificación del rol externo del modificador con el rol referencial del elemento al que modifica, con lo que su definición también es válida para todos los tipos de modificación, y no sólo para la verbal. Maienborn se ocupa en su investigación fundamentalmente de los 
locativos, a los que define como predicados de primer orden sobre objetos y situaciones que denotan la propiedad de estar localizado en una cierta región espacial, independientemente de su posición en la oración.

Hay que partir por lo tanto de la existencia de distintos tipos de "apariciones" a las que se puede clasificar como modificadores, que operan desde distintos niveles sobre distintas entidades, aunque en estas líneas sólo se va a tratar la modificación verbal.

Es necesario en este punto aclarar la manera en que vamos a utilizar el término modificación verbal: bajo nuestro punto de vista, los modificadores verbales son elementos que están presentes (aunque sea de manera potencial) en la órbita semántica de los predicados verbales. Desde aquí modifican a unos u otros elementos con un mayor o menor grado de especificidad respecto a lo que se podría llamar el núcleo de la predicación. En la CS y en la SF inciden o bien directamente sobre el núcleo de la predicación, o bien sobre alguna de las proposiciones de las que se compone su significado; otros modales inciden sobre el conjunto semántico formado por el núcleo unido a alguno de sus slots argumentales, y finalmente otros lo hacen sobre la situación a la que se hace referencia en un uso concreto del verbo.

La modificación es, por lo tanto, un fenómeno que puede afectar directamente a este núcleo y puede tener repercusiones en la estructura interna y configuración de la predicación en los niveles conceptual y semántico. Se realiza en la superficie casi siempre en forma de adverbios o grupos de palabras (generalmente PP) de significado equivalente, aunque en algunos casos (como algunos instrumentales 0 temporales) pueden aparecer también como grupos nominales sin preposición.

Pensamos que los modales, locales y temporales se encuentran presentes en la semántica del verbo en forma de modificadores y no se deben dejar de lado en la teoría de valencias, de la que no se debe olvidar que se concibió orientada a la didáctica de lenguas. Los modales los consideramos los modificadores más próximos al predicado constructor, en el sentido de que son (o pueden ser) más específicos; los locales y temporales también los sitúo en este nivel, un poco más alejados que los anteriores del predicado, ya que son menos específicos y más libres en cuanto a sus posibilidades de combinación. Siguiendo este criterio serían los de tiempo los que ofrecen menos restricciones para su combinación con predicados verbales, por tanto, los menos específicos.

\subsection{DISTINCIÓN ENTRE MODIFICADORES/ADJUNTOS}

Es importante en el modelo la distinción entre argumentos/complementos y modificadores/adjuntos. A pesar de pensar que se trata de elementos de la misma naturaleza ontológica, tiene sentido llevar a cabo esta distinción, que hemos adoptado de Wotjak (1984), pero que también emplea Steinitz (1997), ya que existen elementos que aparecen en la descomposición del significado de los verbos 
en forma de argumentos o modificadores pero nunca pasan a la superficie. Con los argumentos, el ejemplo más claro es el caso del situacional, que sólo aparece en los análisis conceptual y semántico, pero nunca llega a "materializarse". Igualmente ocurre con algunos modificadores, que nunca llegan a pasar a la superficie en forma de unidades léxicas. Por lo tanto, la correspondencia de los modificadores a nivel oracional son los adjuntos.

\subsection{CLASIFICACIÓN DE LOS MODIFICADORES VERBALES}

Según lo explicado y tomando como criterio la incidencia y esa hipotética distancia respecto al núcleo de la predicación, distinguimos los siguientes tipos de modificadores:

- Los modificadores que afectan al núcleo de la predicación: son los que T. Kotschi (1991: 130) llama $K$-Zirkumstanten ( $K=N u ́ c l e o)$. Siguiendo a A. J. Greimas (1966), los denominamos modificadores/semas latentes. Como característica distintiva de estos modificadores hay que señalar que al especificarlos dan lugar a la creación de unidades léxicas nuevas. Pueden ser:

1) Modales: como es el caso de <velocidad> en los verbos de movimiento: andar, correr, pasear, etc.

2) Locales: como es el caso del <medio> también en verbos de movimiento: $i r$, andar, nadar, volar, bucear, etc.

3) Temporales: como <tiempo> en los verbos de cambio de posesión: dar, regalar, prestar, etc.

- Los modificadores modales que afectan a la estructura semántica formada por la unión del núcleo de la predicación a uno de sus argumentos, preferentemente al sujeto u objeto directo. Esta estructura, y en consecuencia estos elementos, se denominan modificadores del nudo actancial, conservando el término que introdujo L. Melis (1983). Pueden ser:

- Los que se refieren al nudo actancial sujeto-verbo caracterizan el modo de actuación o participación del sujeto, que tiene que pertenecer a las categorías [+ANLM], [+/- HUM]. Ejemplos:

4) Pierre a renversé les voitures/Pierre a furieusement renversé les voitures

4a) Le choc a renversé les voitures/*Le choc a furieusement renversé les voitures (Melis, 1983: 44)

5) Marcos perdió el autobús deliberadamente para no cenar aquí (Cuartero, 2000: 76)

5a) *El árbol perdió las hojas deliberadamente.

- Los que están relacionados con el nudo verbo-objeto tampoco se pueden combinar en principio con verbos de estado, tiene que estar presente un sujeto $<$ AGENS>, <EXPERIENCER> o <CAUSATIVO> en el sentido de Cuartero (2000), aunque no afectan en ningún caso a éste, sino al OD. 
6) Il a lancé le projectile d'un movement violent / á l'aide d'une catapulte

6a) *Il a lancé cette idée d'un movement violent / ál'aide d'une catapulte

7) Sophie y Simon usan palabras incongruentemente

7a) *Sophie y Simon usan juguetes incongruentemente.

- Otro caso de incidencia sobre este nudo actancial es el de algunos adverbios, que quizás por causa de frecuentes contactos con una estructura de este tipo, han estrechado hasta tal extremo su unión, que son difícilmente intercambiables de un predicado a otro sin que se aprecien modificaciones en su significado (o en su incidencia). Pueden incidir sobre la estructura de nudo actancial en sus dos variedades así como exclusivamente sobre el predicado en cuestión.

8) Das Essen war scharf gewürzt

8a) Hast du das Messer scharf gemacht?

8b)Bevor die Rakete abgeschossen wird, macht der Pilot sie scharf

3) Los modificadores que afectan a la situación a la que se hace referencia. Siguiendo a Maienborn (1998), dentro de éstos hay también dos grupos:

- Modificadores internos a la situación (situation-internal modifiers), función que desempeñan los locales (en el estadio actual de nuestras investigaciones no hemos encontrado ejemplos de otros tipos de modificadores). Lo que sí se aprecia es cierta incidencia de éstos sobre el verbo y el OD, a la manera de ciertos (modales) de nudo actancial. A pesar de ser locales, tienen un cierto matiz modal. Expresan una relación espacial que se da dentro de la situación a la que hace referencia el verbo (Maienborn, 1998; pág. 4):

9) Der Koch hat das Hähnchen in einer Marihuana-Tunke zubereitet

10) Domingo ha leído la noticia en el periódico.

- Modificadores externos a la situación (situation-external modifiers), que localizan la situación como un todo, independientemente de su organización interna (Maienborn, 1998: 3). Es un tipo de incidencia que también se adscribe a las 3 categorías.

11) Modo: Gonzalo compró la casa rápido

12) Lugar: Gonzalo compró la casa en Chipiona

13) Tiempo: Gonzalo compró la casa en 1999.

En su artículo, Maienborn afirma que éste es el comportamiento estándar de los modificadores locales, y nosotros creemos que esta afirmación se puede ampliar también a los otros dos tipos que nos ocupan. De esta manera, podemos afirmar que ésta es la función canónica de los modificadores verbales en el sentido propuesto.

\section{BIBLIOGRAFIA}

Bondzio, W. (1971), Valenz, Bedeutung und Satzmodelle, en G. Helbig (ed.), Beiträge zur Valenztheorie. VEB Max Niemeyer Verlag, Halle, 85-103.

Bondzio, W. (1974), Die Valenz zweiter Stufe als Grundlage der Adverbialsyntax. WZHUB, Ges.Sprachw. R. XXIII, 3/4, 245-256. 
Bondzio, W., Göllmer, B. (1976), Wortbedeutung und syntaktische Realisierungsmodelle Materialien zur semantischen Valenztheorie. WZHUB, Ges.-Sprachw. R XXV, 6, 699-706.

Cuartero Otal, J. (2000), Algunas consideraciones sobre la agentividad en español. Anuario de Estudios Filológicos, XXIII, 65-76.

Greimas, A. J. (1966), Sémantique structurale; recherche de méthode. Paris: Librairic Larousse.

Kotschi, Th. (1991), Zirkumstanten und komplexe Prädikate, en P. Koch y Th. Krefeld (eds.), Connexiones Romanicae: Dependenz und Valenz in romanischen Sprachen. Tübingen: Max Niemeyer Verlag, 129-137.

Maienborn, C. (1990), Position und Bewegung: Zur Semantik lokaler Verben. IWBS Report 138, Universität Hamburg.

Maienborn, C. (1998), The Grammar and Pragmatics of Locative Modifiers. Manuscrito. Berlin: Humboldt Universităt.

Meinhard, H.J. (1994), Plädoyer fïr das Konzept der Valenz zweiter Stufe-und fïr seine Revision, en W. Thielemann y K. Welke (Eds.), Valenztheorie - Werden und Wirkung. Wilhelm Bondzio zum 65. Geburtstag. Münster: Nodus Verlag, 71-76.

Melis, L. (1983), Les circonstants et la phrase: étude sur la classification et la systématique des compléments circonstanciels en francais moderne. Louvain: Presses Universitaires de Louvain.

Steinit z, R. (1997), Valenznotwendige Präpositionalphrasen: weder Argument- noch Adjunktposition, en Christa Dürscheid et al. (eds.), Festschrift für Heinz Vater zum 65. Geburtstag. Tibingen: Max Niemeyer Verlag, 329-350.

Wotjak, G. (1984), Zur Aktantifizierung von Argumenten ausgewählter deutscher Verben. Zeitschrift fuir Germanistik 4, 401-414. 